\title{
Quantum teleportation of four-dimensional qudits
}

\author{
M. Al-Amri, ${ }^{1,2, *}$ Jörg Evers, ${ }^{2,3, \dagger}$ and M. Suhail Zubairy ${ }^{2,3, \ddagger}$ \\ ${ }^{1}$ The National Center for Mathematics and Physics, KACST, P.O. Box 6086, Riyadh 11442, Saudi Arabia \\ ${ }^{2}$ Max-Planck-Institut für Kernphysik, Saupfercheckweg 1, D-69117 Heidelberg, Germany \\ ${ }^{3}$ Institute for Quantum Studies and Department of Physics and Astronomy, Texas A\&M University, College Station, Texas 77843-4242, USA
}

(Received 11 May 2010; published 25 August 2010)

\begin{abstract}
A protocol for the teleportation of arbitrary quantum states of four-dimensional qudits is presented. The qudit to be teleported is encoded in the combined state of two ensembles of atoms placed in a cavity at the sender's side. The receiver uses a similar setup, with his atoms prepared in a particular initial state. The teleportation protocol then consists of adiabatic mapping of the ensemble states onto photonic degrees of freedom, which are then directed onto a specific beam splitter and detection setup. For part of the measurement outcome, the qudit state is fully transferred to the receiver. Other detection events lead to partial teleportation or failed teleportation attempts. The interpretation of the different detection outcomes and possible ways of improving the full teleportation probability are discussed.
\end{abstract}

DOI: 10.1103/PhysRevA.82.022329

PACS number(s): 03.67.Hk, 03.67.Lx, 42.50.Ex

\section{INTRODUCTION}

Quantum teleportation is a basic ingredient in quantuminformation architectures [1,2]. It makes it possible to transfer faithfully an unknown quantum state of one quantum system to another quantum system, independent of the spatial separation between the two quantum systems. Teleportation relies on the establishment of entanglement between the two communication sites and on a classical communication channel that makes it possible to compare measurement outcomes of sender and receiver. It was discovered in 1993 [3], and since then, several teleportation schemes have been suggested [4-7]. Experiments have been performed, such as between photonic qubits [8-14], photons and a single atomic qubit $[15,16]$, and two atomic qubits [17-19].

So far, however, teleportation schemes have focused on the transfer of qubits, that is, information carriers of the type

$$
\left|\Psi_{2}\right\rangle=\alpha|0\rangle+\beta|1\rangle,
$$

encoded as a superposition of two basis states $|0\rangle$ and $|1\rangle$. An extension of this is the teleportation of Dicke-type states encoded in two atoms $[20,21]$. These three-dimensional Dicke states are of the form

$$
\left|\Psi_{3}\right\rangle=\alpha|00\rangle+\beta \frac{|01\rangle+|10\rangle}{\sqrt{2}}+\gamma|11\rangle .
$$

Here we go one step further and focus on the teleportation of four-dimensional qudits,

$$
\left|\Psi_{4}\right\rangle=\alpha|0\rangle+\beta|1\rangle+\gamma|2\rangle+\delta|3\rangle .
$$

It is important to note that $\left|\Psi_{4}\right\rangle$ cannot merely be represented by a combination of two qubit states $\left|\Psi_{2}\right\rangle$. The four coefficients in $\left|\Psi_{4}\right\rangle$ contain eight real degrees of freedom (DOF), constrained by the normalization and the overall phase, which leaves six DOF. Two qubit states also have four coefficients with eight DOF, but each qubit is constrained by an overall

\footnotetext{
*mdalamri@kacst.edu.sa

†joerg.evers@mpi-hd.mpg.de

${ }^{\ddagger}$ zubairy@physics.tamu.edu
}

phase and an overall normalization, such that the number of DOF is lower. Since higher-order qudits are an important resource in quantum-information processing [22], a teleportation scheme for such more complex information carriers is certainly desirable. Teleportation of higher-dimensional quantum states have been considered before in a cavity-QED setting [23] and in a solid-state setup [24]. The teleportation of a subset of four-dimensional photonic two-qubit states has been reported in [25].

In the present scheme, the initial unknown qudit state is encoded in two ensembles of atoms which are placed in a cavity at the sender's (Alice's) side [20,21] (see Fig. 1). Coherent light fields can be used to entangle atomic ensembles $[26,27]$. Bob, the receiver, has a similar setup and prepares his ensembles in a specific initial state. The atomic excitation is then coherently mapped onto photonic DOF, for example, by means of adiabatic passage [15,16,28-31]. The produced photons are combined in a specific detection setup, leading to three classes of detection events. We find that one class of detection events, which occurs with $25 \%$ probability, leads to a full teleportation of the qudit state from Alice to Bob. The second class leads to a partial transfer of the initial state. The third class contains failed teleportation events. We interpret the different detection outcomes and analyze possibilities of improving the probability for full teleportation events. One interesting feature of our setup is that the intermediate photonic entangled state is a superposition of different energy states. This, on the one hand, leads to a restriction on the acceptable path-length difference for photons coming from sender and receiver, respectively, but at the same time distinguishes our approach from most other entanglement setups.

The article is organized as follows. In Sec. II, the teleportation scheme is presented. The initial preparation is discussed in Sec. II A, the detection setup in Sec. II B, and the different detection outcomes in Sec. II C. Finally, Sec. III discusses and summarizes our results.

\section{TELEPORTATION SCHEME}

Our goal is to teleport a quantum state encoded in two separate ensembles of atoms (see Fig. 1). The two ensembles 


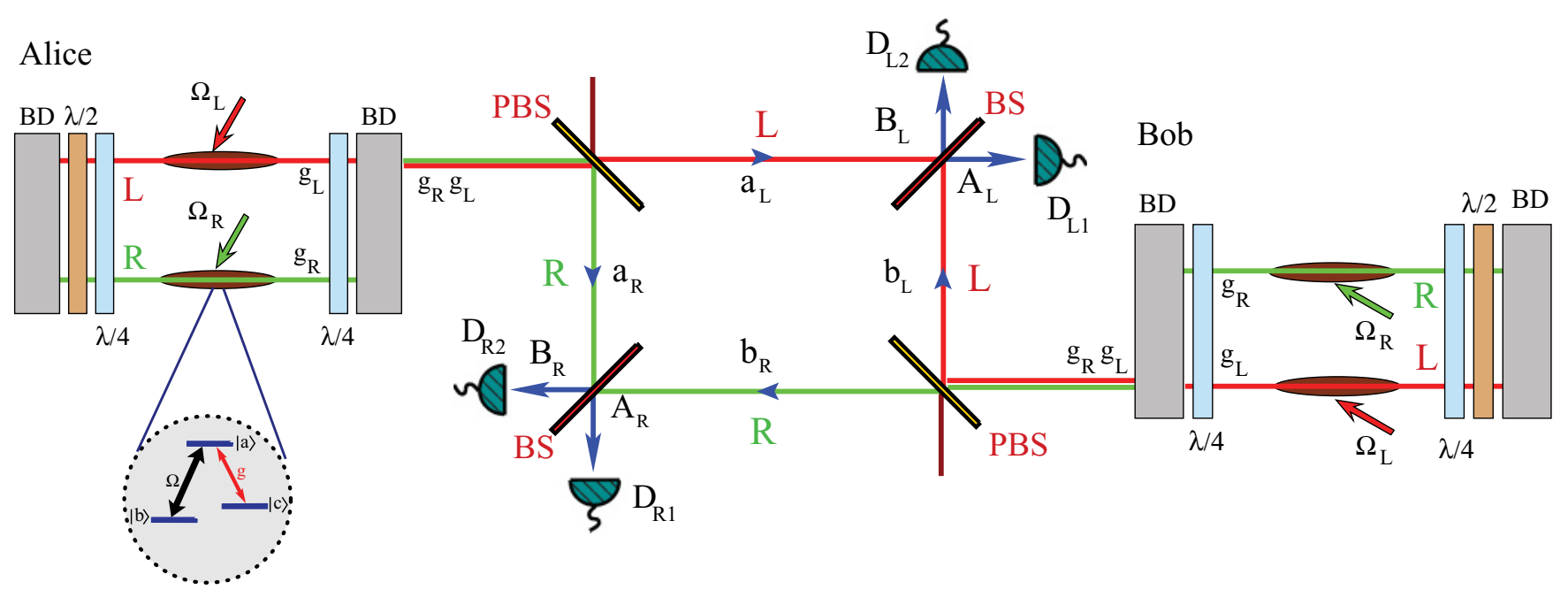

FIG. 1. (Color online) Schematic representation of the teleportation setup. On Alice's side, two ensembles of atoms encode the state to be teleported, and on Bob's side, the ensembles are prepared in a particular initial state in a similar setup. The inset shows the level configuration of each atom in the ensembles. Using adiabatic state transfer, both parties then map the ensemble states coherently to photonic DOF, which enter the beam splitter setup. The setup for the mapping between ensembles and light is similar to the experiment reported in [28]. The initial state prepared by Alice enters in the top-left corner. Bob's state enters in the bottom-right corner. The detectors are placed in the two other corners. The input photon states are passed through polarization beam splitters (PBS) to separate the two photon modes, and the outputs of the PBSs are recombined on beam splitters (BS). The output modes of the beam splitters are detected by four photon detectors $D_{L_{1}}, D_{L_{2}}, D_{R_{1}}$, and $D_{R_{2}}$.

are labeled $L$ and $R$, respectively, and consist of three-level atoms in $\Lambda$-type configuration, with excited state $|a\rangle$ and degenerate ground states $|b\rangle$ and $|c\rangle$. The two ensembles are spatially separated within the same cavity, such that they can be addressed individually by laser fields. Each cavity is such that inside, the ensembles experience the same field polarization, whereas outside the cavity, the two light fields interacting with the ensembles have different polarizations and thus are distinguishable [28]. The state to be teleported is encoded in the atomic ground states and can be written as

$$
\begin{aligned}
|\psi\rangle_{A}^{\text {in }}= & C_{0}^{A}\left|c_{L} c_{R}\right\rangle^{A}+C_{1}^{A}\left|b_{L} c_{R}\right\rangle^{A}+C_{2}^{A}\left|c_{L} b_{R}\right\rangle^{A} \\
& +C_{3}^{A}\left|b_{L} b_{R}\right\rangle^{A},
\end{aligned}
$$

with normalization $\sum_{i}\left|C_{i}^{A}\right|^{2}=1$. A state of this type could be prepared by first generating four-dimensional entangled photon states [32-35] and then mapping this state into the ensemble using adiabatic passage. Alternatively, a direct preparation in the atomic ensembles could be assisted, for example, by the Rydberg blockade mechanism [36-38].

\section{A. Initial preparation}

The first step is to coherently map the atomic state Eq. (4) onto photonic DOF. For this, we make use of the well-known technique of adiabatic passage $[28,39]$ (Note that there are also non-adiabatic methods to prepare atom entanglement across cavities [40], and to teleport photonic entanglement onto atomic ensembles [41]). By applying suitable control fields to the ensembles, the following mapping can be achieved:

$$
\begin{aligned}
& \left|c_{L} c_{R}\right\rangle^{A}\left|0_{L} 0_{R}\right\rangle^{A} \longrightarrow\left|c_{L} c_{R}\right\rangle^{A}\left|0_{L} 0_{R}\right\rangle^{A}, \\
& \left|b_{L} c_{R}\right\rangle^{A}\left|0_{L} 0_{R}\right\rangle^{A} \longrightarrow\left|c_{L} c_{R}\right\rangle^{A}\left|1_{L} 0_{R}\right\rangle^{A},
\end{aligned}
$$

$$
\begin{aligned}
& \left|c_{L} b_{R}\right\rangle^{A}\left|0_{L} 0_{R}\right\rangle^{A} \longrightarrow\left|c_{L} c_{R}\right\rangle^{A}\left|0_{L} 1_{R}\right\rangle^{A}, \\
& \left|b_{L} b_{R}\right\rangle^{A}\left|0_{L} 0_{R}\right\rangle^{A} \longrightarrow\left|c_{L} c_{R}\right\rangle^{A}\left|1_{L} 1_{R}\right\rangle^{A} .
\end{aligned}
$$

In this way, the ensemble state is mapped onto the cavity field state:

$$
\begin{aligned}
|\psi\rangle_{A}= & \left(C_{0}^{A}\left|0_{L} 0_{R}\right\rangle^{A}+C_{1}^{A}\left|1_{L} 0_{R}\right\rangle^{A}+C_{2}^{A}\left|0_{L} 1_{R}\right\rangle^{A}\right. \\
& \left.+C_{3}^{A}\left|1_{L} 1_{R}\right\rangle\right)\left|c_{L} c_{R}\right\rangle^{A} .
\end{aligned}
$$

Note that by making use of a setup suggested in [28], the two photonic DOF leaving the cavity have different polarization and spatially overlap, even though inside the cavity both ensembles are in separate locations and experience cavity fields with the same polarization. This is crucial, as otherwise the ensemble state after the mapping to the photonic DOF does not factorize out. Furthermore, the intermediate photonic state in Eq. (6) is a superposition of different energy states. This means that due to the free time evolution throughout the propagation of the photons, the state amplitudes will acquire different, time-dependent relative phases. In the following, we, for simplicity, assume that the path lengths for photons from Alice and Bob to the detectors coincide and neglect overall phase factors to simplify the notation. In Sec. III, the path-length difference is discussed in more detail.

On the receiver side, we assume that Bob prepares his ensembles in the state $\left|b_{L} b_{R}\right\rangle$, which is possible since the distant preparation of the ensembles allows for individual addressing by laser fields. Then, similar to Alice, Bob uses the coherent mapping between the ensemble state and his output light field modes in Eqs. (5) to obtain the state

$$
\begin{aligned}
|\psi\rangle_{B}= & D_{0}^{B}\left|b_{L} b_{R}\right\rangle^{B}\left|0_{L} 0_{R}\right\rangle^{B}+D_{1}^{B}\left|c_{L} b_{R}\right\rangle^{B}\left|1_{L} 0_{R}\right\rangle^{B} \\
& +D_{2}^{B}\left|b_{L} c_{R}\right\rangle^{B}\left|0_{L} 1_{R}\right\rangle^{B}+D_{3}^{B}\left|c_{L} c_{R}\right\rangle^{B}\left|1_{L} 1_{R}\right\rangle^{B} .
\end{aligned}
$$


The joint state of Alice's and Bob's systems after the mapping onto the photonic modes is given by

$$
|\psi\rangle_{A B}=|\psi\rangle_{A} \otimes|\psi\rangle_{B},
$$

and the explicit form is given in Appendix A.

\section{B. Photon detection setup}

We now turn to the beam splitter setup shown in Fig. 1. Alice's photonic state enters the setup on the top-left corner, and a polarizing beam splitter (PBS) is used to separate the two field modes $a_{L}$ and $a_{R}$. Similarly, Bob's state enters in the bottom-right corner and is separated using a PBS into $b_{L}$ and $b_{R}$. The resulting modes are recombined on two beam splitters (BS) in the top right and bottom left corner, which together have four output modes $A_{L}, B_{L}, A_{R}$, and $B_{R}$ that are detected by the detectors $D$. To analyze the operation of this setup, we now calculate the wave functions after a certain detection event has taken place. For this, we make use of the beam-splitter relations [42],

$$
\begin{aligned}
& \hat{A}_{R}=t_{R} \hat{a}_{R}+r_{R} \hat{b}_{R}, \\
& \hat{B}_{R}=r_{R} \hat{a}_{R}-t_{R} \hat{b}_{R}, \\
& \hat{A}_{L}=t_{L} \hat{a}_{L}+r_{L} \hat{b}_{L}, \\
& \hat{B}_{L}=r_{L} \hat{a}_{L}-t_{L} \hat{b}_{L},
\end{aligned}
$$

where $\hat{a}_{R}$ and $\hat{b}_{R}$ are the destruction operators for right input modes, $\hat{A}_{R}$ and $\hat{B}_{R}$ the destruction operators for the right output modes (and analogously for the $L$ modes). The beam splitter reflectivities $r_{i}$ and transmittivities $t_{i}(i \in\{L, R\})$ are real and positive and satisfy $r_{R}^{2}+t_{R}^{2}=1$ and $r_{L}^{2}+t_{L}^{2}=1$.

As an example for the calculation, we demonstrate how one possible photonic input state is transformed by the beam splitters:

$$
\begin{aligned}
\left|0_{L} 1_{R}\right\rangle^{A}\left|1_{L} 0_{R}\right\rangle^{B}= & a_{R}^{\dagger} b_{L}^{\dagger}|\mathrm{vac}\rangle \\
= & \left(t_{R} A_{R}+r_{R} B_{R}\right)\left(r_{L} A_{L}-t_{L} B_{L}\right)|\mathrm{vac}\rangle \\
= & t_{R} r_{L}|10,10\rangle-t_{R} t_{L}|01,10\rangle \\
& +r_{R} r_{L}|10,01\rangle-r_{R} t_{L}|01,01\rangle,
\end{aligned}
$$

where $\left|A_{L} B_{L}, A_{R} B_{R}\right\rangle$ denotes the number of photons in the four output modes $A_{L}, B_{L}, A_{R}$, and $B_{R}$ after the beam splitters, and $|\mathrm{vac}\rangle$ is the vacuum state.

\section{Detection events}

We now analyze the possible detection events. After each detection event, the total system wave function collapses to the state $\left|\psi_{A_{L} B_{L}, A_{R} B_{R}}\right\rangle$ given by

$$
\left|\psi_{A_{L} B_{L}, A_{R} B_{R}}\right\rangle=\left\langle A_{L} B_{L}, A_{R} B_{R} \mid \psi\right\rangle_{A B} .
$$

A list of all possible detection events is provided in Appendix B. A careful analysis of these events reveals that the events can be classified into three categories. The first category encompasses events which lead to a teleportation of the full initial state from Alice to Bob and in this sense are the most favorable for teleportation. We denote these events as full teleportation events. The second class leads to a partial teleportation in the sense that only two of the four amplitudes of the initial state at Alice's side are transferred to Bob. The third class contains failed teleportation events which do not transfer useful information to Bob.

\section{Full teleportation}

It turns out that for the purpose of teleportation, the detection events with one photon detected in either of the two left detectors and one photon in either of the two right detectors are the most favorable. Their collapsed wave functions receive contributions from the following parts of Eq. (8) [see also Eq. (A1)]:

$$
\begin{aligned}
& C_{0}^{A} D_{3}^{B}\left|c_{L} c_{R}\right\rangle^{B}\left|0_{L} 0_{R}\right\rangle^{A}\left|1_{L} 1_{R}\right\rangle^{B} \\
&= C_{0}^{A} D_{3}^{B}\left|c_{L} c_{R}\right\rangle\left\{r_{L} r_{R}|10,10\rangle-r_{L} t_{R}|10,01\rangle\right. \\
&\left.\quad-t_{L} r_{R}|01,10\rangle+t_{L} t_{R}|01,01\rangle\right\}, \\
& C_{1}^{A} D_{2}^{B}\left|b_{L} c_{R}\right\rangle^{B}\left|1_{L} 0_{R}\right\rangle^{A}\left|0_{L} 1_{R}\right\rangle^{B}=C_{1}^{A} D_{2}^{B}\left|b_{L} c_{R}\right\rangle\left\{t_{L} r_{R}|10,10\rangle-t_{L} t_{R}|10,01\rangle\right. \\
&\left.\quad+r_{L} r_{R}|01,10\rangle-r_{L} t_{R}|01,01\rangle\right\}, \\
& C_{2}^{A} D_{1}^{B}\left|c_{L} b_{R}\right\rangle^{B}\left|0_{L} 1_{R}\right\rangle^{A}\left|1_{L} 0_{R}\right\rangle^{B} \\
&= C_{2}^{A} D_{1}^{B}\left|c_{L} b_{R}\right\rangle\left\{r_{L} t_{R}|10,10\rangle+r_{L} r_{R}|10,01\rangle\right. \\
&\left.\quad-t_{L} t_{R}|01,10\rangle-t_{L} r_{R}|01,01\rangle\right\}, \\
& C_{3}^{A} D_{0}^{B}\left|b_{L} b_{R}\right\rangle^{B}\left|1_{L} 1_{R}\right\rangle^{A}\left|0_{L} 0_{R}\right\rangle^{B} \\
&= C_{3}^{A} D_{0}^{B}\left|b_{L} b_{R}\right\rangle\left\{t_{L} t_{R}|10,10\rangle+t_{L} r_{R}|10,01\rangle\right. \\
&\left.+r_{L} t_{R}|01,10\rangle+r_{L} r_{R}|01,01\rangle\right\},
\end{aligned}
$$

where we have transformed the input photon modes into the output modes as in Eq. (10). Grouping these results according to the different detection events, we obtain

$$
\begin{aligned}
\left|\psi_{10,10}\right\rangle= & C_{3}^{A} D_{0}^{B} t_{L} t_{R}\left|b_{L} b_{R}\right\rangle^{B}+C_{1}^{A} D_{2}^{B} r_{R} t_{L}\left|b_{L} c_{R}\right\rangle^{B} \\
& +C_{2}^{A} D_{1}^{B} r_{L} t_{R}\left|c_{L} b_{R}\right\rangle^{B}+C_{0}^{A} D_{3}^{B} r_{L} r_{R}\left|c_{L} c_{R}\right\rangle^{B}, \\
\left|\psi_{01,10}\right\rangle= & C_{3}^{A} D_{0}^{B} r_{L} t_{R}\left|b_{L} b_{R}\right\rangle^{B}+C_{1}^{A} D_{2}^{B} r_{L} r_{R}\left|b_{L} c_{R}\right\rangle^{B} \\
& -C_{2}^{A} D_{1}^{B} t_{L} t_{R}\left|c_{L} b_{R}\right\rangle^{B}-C_{0}^{A} D_{3}^{B} t_{L} r_{R}\left|c_{L} c_{R}\right\rangle^{B}, \\
\left|\psi_{10,01}\right\rangle= & C_{3}^{A} D_{0}^{B} r_{R} t_{L}\left|b_{L} b_{R}\right\rangle^{B}-C_{1}^{A} D_{2}^{B} t_{L} t_{R}\left|b_{L} c_{R}\right\rangle^{B} \\
& +C_{2}^{A} D_{1}^{B} r_{L} r_{R}\left|c_{L} b_{R}\right\rangle^{B}-C_{0}^{A} D_{3}^{B} r_{L} t_{R}\left|c_{L} c_{R}\right\rangle^{B}, \\
& \\
\left|\psi_{01,01}\right\rangle= & C_{3}^{A} D_{0}^{B} r_{L} r_{R}\left|b_{L} b_{R}\right\rangle^{B}-C_{1}^{A} D_{2}^{B} r_{L} t_{R}\left|b_{L} c_{R}\right\rangle^{B} \\
& -C_{2}^{A} D_{1}^{B} r_{R} t_{L}\left|c_{L} b_{R}\right\rangle^{B}+C_{0}^{A} D_{3}^{B} t_{L} t_{R}\left|c_{L} c_{R}\right\rangle^{B} .
\end{aligned}
$$

To simplify these expressions and to allow for a straightforward interpretation, we specialize to the case of balanced beam splitters $t_{L}=t_{R}=r_{L}=r_{R}=1 / \sqrt{2}$ and balanced preparation of the initial state on Bob' side, $D_{i}^{B}=1 / \sqrt{4}$. Then

$$
\begin{aligned}
\left|\psi_{10,10}\right\rangle= & \frac{1}{4}\left(C_{3}^{A}\left|b_{L} b_{R}\right\rangle^{B}+C_{1}^{A}\left|b_{L} c_{R}\right\rangle^{B}\right. \\
& \left.+C_{2}^{A}\left|c_{L} b_{R}\right\rangle^{B}+C_{0}^{A}\left|c_{L} c_{R}\right\rangle^{B}\right), \\
\left|\psi_{01,10}\right\rangle= & \frac{1}{4}\left(C_{3}^{A}\left|b_{L} b_{R}\right\rangle^{B}+C_{1}^{A}\left|b_{L} c_{R}\right\rangle^{B}\right. \\
& \left.-C_{2}^{A}\left|c_{L} b_{R}\right\rangle^{B}-C_{0}^{A}\left|c_{L} c_{R}\right\rangle^{B}\right), \\
\left|\psi_{10,01}\right\rangle= & \frac{1}{4}\left(C_{3}^{A}\left|b_{L} b_{R}\right\rangle^{B}-C_{1}^{A}\left|b_{L} c_{R}\right\rangle^{B}\right. \\
& \left.+C_{2}^{A}\left|c_{L} b_{R}\right\rangle^{B}-C_{0}^{A}\left|c_{L} c_{R}\right\rangle^{B}\right),
\end{aligned}
$$




$$
\begin{aligned}
\left|\psi_{01,01}\right\rangle= & \frac{1}{4}\left(C_{3}^{A}\left|b_{L} b_{R}\right\rangle^{B}-C_{1}^{A}\left|b_{L} c_{R}\right\rangle^{B}\right. \\
& \left.-C_{2}^{A}\left|c_{L} b_{R}\right\rangle^{B}+C_{0}^{A}\left|c_{L} c_{R}\right\rangle^{B}\right) .
\end{aligned}
$$

It can be seen that up to a local unitary transformation (by which we mean operations acting on both ensembles at Bob's side only) changing the relative sign of the different addends, all four detection events lead to a complete teleportation of the original input state [Eq. (4)] from Alice to Bob. This teleportation of the full quantum state is possible since the collapsed wave functions in Eqs. (14) are coherent superpositions of all four possible atomic states at Bob's side.

Overall, the probability for a full teleportation is $25 \%$, as long as the initial state on Bob's side is prepared balanced, that is, $D_{i}^{B}=1 / \sqrt{4}$ for $i \in\{1, \ldots, 4\}$, but independent of the choice of the beam-splitter parameters. That means that in one quarter of the teleportation attempts, the full four-level state is transferred with certainty to Bob.

\section{Partial teleportation}

In a second class of detection events, the wave function collapses to a superposition of two atomic states on Bob's side upon detection. These are the events with only one photon in either of the four detectors and the events with two photons in one mode of the left detector and one photon in a mode of the right detector or vice versa. In total, there are 12 detection events leading to partial teleportation for the case of balanced beam splitters. Two examples of these events are

$$
\begin{aligned}
\left|\psi_{10,00}\right\rangle & =\frac{1}{2 \sqrt{2}}\left(C_{0}^{A}\left|c_{L} b_{R}\right\rangle^{B}+C_{1}^{A}\left|b_{L} b_{R}\right\rangle^{B}\right), \\
\left|\psi_{10,02}\right\rangle & =-\frac{1}{4}\left(C_{2}^{A}\left|c_{L} c_{R}\right\rangle^{B}+C_{3}^{A}\left|b_{L} c_{R}\right\rangle^{B}\right),
\end{aligned}
$$

and the remaining cases are enlisted in Appendix B. It can be seen that in these events, only two out of the four amplitudes in the original input state [Eq. (4)] are teleported, such that the teleportation is partially successful. For example, in Eq. (15a), the left ensemble at Bob's side is projected onto the state $\left(C_{0}^{A}|c\rangle+C_{1}^{A}|b\rangle\right) /(2 \sqrt{2})$, whereas in Eq. (15b), Bob's left ensemble assumes the final state $-\left(C_{2}^{A}|c\rangle+C_{3}^{A}|b\rangle\right) / 4$.

The usefulness of these teleportation events depends on the desired application. In terms of a teleportation of the full initial state Eq. (4), they are of little help since two partial teleportations cannot be combined to give a full teleportation. For example, even though the two partial teleportation events in Eq. (15) together contain all four amplitudes, the two events cannot be combined to the original state Eq. (4), since the phase between the two partially teleported states is unknown.

However, for other applications, knowledge of two of the amplitudes could be sufficient, or it could be possible to combine the outcomes of several partial teleportations to form a new state different from Eq. (4), but nevertheless useful. If full teleportation of the initial state is desired, then it might be possible to apply the intermittent partial teleportations to correct for inevitable errors using a postprocessing scheme at Bob's side.

In total, partial teleportations occur with half probability for balanced input state at Bob's side.

\section{Failed teleportation}

The third class of detection events leads to collapsed wave functions which contain a single state of Bob's atoms. Two examples for these events are

$$
\begin{gathered}
\left|\psi_{00,00}\right\rangle=\frac{1}{\sqrt{4}} C_{0}^{A}\left|b_{L} b_{R}\right\rangle^{B}, \\
\left|\psi_{20,20}\right\rangle=\frac{1}{4} C_{3}^{A}\left|c_{L} c_{R}\right\rangle^{B} .
\end{gathered}
$$

Failed teleportations occur in $25 \%$ of the teleportation attempts for balanced input state at Bob's side, independent of the beamsplitter parameters.

\section{DISCUSSION AND SUMMARY}

In summary, we have presented a teleportation scheme to transfer an arbitrary quantum state of the form

$$
\begin{aligned}
|\psi\rangle_{A}^{\text {in }}= & C_{0}^{A}\left|c_{L} c_{R}\right\rangle^{A}+C_{1}^{A}\left|b_{L} c_{R}\right\rangle^{A} \\
& +C_{2}^{A}\left|c_{L} b_{R}\right\rangle^{A}+C_{3}^{A}\left|b_{L} b_{R}\right\rangle^{A}
\end{aligned}
$$

from the sender to a receiver. Full teleportation of the initial state is achieved in $25 \%$ of the teleportation attempts. The reason for this limited success probability is the fact that due to the specific mapping of the ensemble states onto photonic DOF, the number of photons in the system can be any value from zero to four. Full transfer, however, only occurs if exactly one photon is detected in one of the left detectors and one in either of the right detectors. The probability for full teleportations can be improved if additional constraints apply to the initial state to be teleported at Alice's side. The probability, in general, evaluates to

$$
\begin{aligned}
p_{\text {full }}= & \left|C_{3}^{A}\right|^{2}\left|D_{0}^{B}\right|^{2}+\left|C_{2}^{A}\right|^{2}\left|D_{1}^{B}\right|^{2} \\
& +\left|C_{1}^{A}\right|^{2}\left|D_{2}^{B}\right|^{2}+\left|C_{0}^{A}\right|^{2}\left|D_{3}^{B}\right|^{2},
\end{aligned}
$$

subject to the normalization constraints $\sum_{i}\left|C_{i}^{A}\right|^{2}=1=$ $\sum_{i}\left|D_{i}^{B}\right|^{2}$. If it is known that on average some of the four state amplitudes $C_{i}^{A}$ differ from others, then a suitable choice of imbalance of the parameters $D_{i}^{B}$ can lead to $p_{\text {full }}>$ $25 \%$. For example, assume that on average $\left|C_{0}^{A}\right|^{2}=1 / 4+x$, $\left|C_{1}^{A}\right|^{2}=\left|C_{2}^{A}\right|^{2}=1 / 4$, and $\left|C_{3}^{A}\right|^{2}=1 / 4-x$. Then choosing $\left|D_{0}^{B}\right|^{2}=1 / 4+y,\left|D_{1}^{B}\right|^{2}=\left|D_{2}^{B}\right|^{2}=1 / 4$, and $\left|D_{3}^{B}\right|^{2}=1 / 4-y$ leads to $p_{\text {full }}=1 / 4-2 x y$. Thus, the probability for a full teleportation event can be increased, however, at the cost that the final state at Bob's side needs postprocessing of the state amplitudes. For example, for the preceding nonbalanced parameter choices, the state in Eq. (14a) becomes

$$
\begin{aligned}
\left|\psi_{10,10}\right\rangle= & \frac{1}{4}\left(C_{3}^{A} \sqrt{1+4 y}\left|b_{L} b_{R}\right\rangle^{B}+C_{1}^{A}\left|b_{L} c_{R}\right\rangle^{B}\right. \\
& \left.+C_{2}^{A}\left|c_{L} b_{R}\right\rangle^{B}+C_{0}^{A} \sqrt{1-4 y}\left|c_{L} c_{R}\right\rangle^{B}\right) .
\end{aligned}
$$

The partial teleportation events which occur with $50 \%$ probability, however, can also be of use, depending on the specific desired application. Straightforward recombining of two partial teleportations to give the full initial state, however, is not possible.

The variable number of photons produced in the transfer from the ensemble to photonic DOF inevitably also leads to failed teleportation attempts. For example, if no photon is 
detected, then the wave function collapses to a state proportional to $\left|b_{L} b_{R}\right\rangle$, since the detection event uniquely defines the possible atomic state to a single possibility. Apart from this trivial case, there are other combinations with, for example, three detected photons that uniquely define the atomic states at Bob's side and therefore do not lead to a transfer of useful superposition states from Alice. At the same time, this variable photon number corresponds to a superposition of different energy states. This leads to time-dependent relative phases acquired by the different addends in the superposition states, thereby modifying the encoded state. This is in part remedied by the fact that, for example, the full teleportation events correspond to a collapse of the total wave functions to states with exactly two photonic excitations. If the respective path length of photons from Alice and Bob to the detectors are equal, then the free time evolution leads only to an overall phase modification of the collapsed wave function. However, if the path lengths of Alice and Bob differ by $P$, then relative phases of order $\Phi=\omega P / c$ with $\hbar \omega$ the photon energy and $c$ the speed of light between the addends arise. The requirement of a negligible phase change $\Phi \ll 2 \pi$ then leads to the conditions $\omega \ll 2 \pi c / P$ or $P \ll 2 \pi c / \omega$ on the photon frequency and the maximum path-length difference to ensure a high fidelity of the teleported state.

Our scheme therefore allows to teleport a more general state than conventional single qubit teleportation schemes, at the price of a lower success probability. Improvement of the success probability could be achieved by a better mapping of the ensemble DOF to photonic states, for example, such that always a pair of photons enters the detection scheme.

\section{ACKNOWLEDGMENTS}

This research is supported by a grant from the King Abdul Aziz City for Science and Technology and an NPRP grant from the Qatar National Research Fund. M.S.Z. acknowledges the support of the Alexander von Humboldt Foundation. M.A. and J.E. gratefully acknowledge the hospitality at Texas A\&M University, where part of this work was done.

\section{APPENDIX A: INITIAL STATE}

The explicit form of the initial state Eq. (8) can be written as

$$
\begin{aligned}
|\psi\rangle_{A B}= & C_{0}^{A} D_{0}^{B}\left|b_{L} b_{R}\right\rangle^{B}\left|0_{L} 0_{R}\right\rangle^{A}\left|0_{L} 0_{R}\right\rangle^{B} \\
& +C_{0}^{A} D_{1}^{B}\left|c_{L} b_{R}\right\rangle^{B}\left|0_{L} 0_{R}\right\rangle^{A}\left|1_{L} 0_{R}\right\rangle^{B} \\
& +C_{0}^{A} D_{2}^{B}\left|b_{L} c_{R}\right\rangle^{B}\left|0_{L} 0_{R}\right\rangle^{A}\left|0_{L} 1_{R}\right\rangle^{B} \\
& +C_{0}^{A} D_{3}^{B}\left|c_{L} c_{R}\right\rangle^{B}\left|0_{L} 0_{R}\right\rangle^{A}\left|1_{L} 1_{R}\right\rangle^{B} \\
& +C_{1}^{A} D_{0}^{B}\left|b_{L} b_{R}\right\rangle^{B}\left|1_{L} 0_{R}\right\rangle^{A}\left|0_{L} 0_{R}\right\rangle^{B} \\
& +C_{1}^{A} D_{1}^{B}\left|c_{L} b_{R}\right\rangle^{B}\left|1_{L} 0_{R}\right\rangle^{A}\left|1_{L} 0_{R}\right\rangle^{B} \\
& +C_{1}^{A} D_{2}^{B}\left|b_{L} c_{R}\right\rangle^{B}\left|1_{L} 0_{R}\right\rangle^{A}\left|0_{L} 1_{R}\right\rangle^{B} \\
& +C_{1}^{A} D_{3}^{B}\left|c_{L} c_{R}\right\rangle^{B}\left|1_{L} 0_{R}\right\rangle^{A}\left|1_{L} 1_{R}\right\rangle^{B} \\
& +C_{2}^{A} D_{0}^{B}\left|b_{L} b_{R}\right\rangle^{B}\left|0_{L} 1_{R}\right\rangle^{A}\left|0_{L} 0_{R}\right\rangle^{B} \\
& +C_{2}^{A} D_{1}^{B}\left|c_{L} b_{R}\right\rangle^{B}\left|0_{L} 1_{R}\right\rangle^{A}\left|1_{L} 0_{R}\right\rangle^{B} \\
& +C_{2}^{A} D_{2}^{B}\left|b_{L} c_{R}\right\rangle^{B}\left|0_{L} 1_{R}\right\rangle^{A}\left|0_{L} 1_{R}\right\rangle^{B}
\end{aligned}
$$

$$
\begin{aligned}
& +C_{2}^{A} D_{3}^{B}\left|c_{L} c_{R}\right\rangle^{B}\left|0_{L} 1_{R}\right\rangle^{A}\left|1_{L} 1_{R}\right\rangle^{B} \\
& +C_{3}^{A} D_{0}^{B}\left|b_{L} b_{R}\right\rangle^{B}\left|1_{L} 1_{R}\right\rangle^{A}\left|0_{L} 0_{R}\right\rangle^{B} \\
& +C_{3}^{A} D_{1}^{B}\left|c_{L} b_{R}\right\rangle^{B}\left|1_{L} 1_{R}\right\rangle^{A}\left|1_{L} 0_{R}\right\rangle^{B} \\
& +C_{3}^{A} D_{2}^{B}\left|b_{L} c_{R}\right\rangle^{B}\left|1_{L} 1_{R}\right\rangle^{A}\left|0_{L} 1_{R}\right\rangle^{B} \\
& +C_{3}^{A} D_{3}^{B}\left|c_{L} c_{R}\right\rangle^{B}\left|1_{L} 1_{R}\right\rangle^{A}\left|1_{L} 1_{R}\right\rangle^{B} .
\end{aligned}
$$

\section{APPENDIX B: SUMMARY OF ALL DETECTION EVENTS}

In the following, all possible detection events are listed. Note that for balanced beam splitters, part of the detection events since in this case $r_{L}^{2}=t_{L}^{2}$ and $r_{R}^{2}=t_{R}^{2}$.

\section{Full teleportation}

$$
\begin{aligned}
\left|\psi_{10,10}\right\rangle= & C_{3}^{A} D_{0}^{B} t_{L} t_{R}\left|b_{L} b_{R}\right\rangle^{B}+C_{1}^{A} D_{2}^{B} r_{R} t_{L}\left|b_{L} c_{R}\right\rangle^{B} \\
& +C_{2}^{A} D_{1}^{B} r_{L} t_{R}\left|c_{L} b_{R}\right\rangle^{B}+C_{0}^{A} D_{3}^{B} r_{L} r_{R}\left|c_{L} c_{R}\right\rangle^{B}, \\
\left|\psi_{01,10}\right\rangle= & C_{3}^{A} D_{0}^{B} r_{L} t_{R}\left|b_{L} b_{R}\right\rangle^{B}+C_{1}^{A} D_{2}^{B} r_{L} r_{R}\left|b_{L} c_{R}\right\rangle^{B} \\
& -C_{2}^{A} D_{1}^{B} t_{L} t_{R}\left|c_{L} b_{R}\right\rangle^{B}-C_{0}^{A} D_{3}^{B} t_{L} r_{R}\left|c_{L} c_{R}\right\rangle^{B}, \\
\left|\psi_{10,01}\right\rangle= & C_{3}^{A} D_{0}^{B} r_{R} t_{L}\left|b_{L} b_{R}\right\rangle^{B}-C_{1}^{A} D_{2}^{B} t_{L} t_{R}\left|b_{L} c_{R}\right\rangle^{B} \\
& +C_{2}^{A} D_{1}^{B} r_{L} r_{R}\left|c_{L} b_{R}\right\rangle^{B}-C_{0}^{A} D_{3}^{B} r_{L} t_{R}\left|c_{L} c_{R}\right\rangle^{B}, \\
\left|\psi_{01,01}\right\rangle= & C_{3}^{A} D_{0}^{B} r_{L} r_{R}\left|b_{L} b_{R}\right\rangle^{B}-C_{1}^{A} D_{2}^{B} r_{L} t_{R}\left|b_{L} c_{R}\right\rangle^{B} \\
& -C_{2}^{A} D_{1}^{B} r_{R} t_{L}\left|c_{L} b_{R}\right\rangle^{B}+C_{0}^{A} D_{3}^{B} t_{L} t_{R}\left|c_{L} c_{R}\right\rangle^{B} .
\end{aligned}
$$

\section{Partial teleportation}

$$
\begin{aligned}
& \left|\psi_{00,10}\right\rangle=C_{2}^{A} D_{0}^{B} t_{R}\left|b_{L} b_{R}\right\rangle^{B}+C_{0}^{A} D_{2}^{B} r_{R}\left|b_{L} c_{R}\right\rangle^{B}, \\
& \left|\psi_{00,01}\right\rangle=C_{2}^{A} D_{0}^{B} r_{R}\left|b_{L} b_{R}\right\rangle^{B}-C_{0}^{A} D_{2}^{B} t_{R}\left|b_{L} c_{R}\right\rangle^{B} \text {, } \\
& \left|\psi_{10,00}\right\rangle=C_{1}^{A} D_{0}^{B} t_{L}\left|b_{L} b_{R}\right\rangle^{B}+C_{0}^{A} D_{1}^{B} r_{L}\left|c_{L} b_{R}\right\rangle^{B} \text {, } \\
& \left|\psi_{01,00}\right\rangle=C_{1}^{A} D_{0}^{B} r_{L}\left|b_{L} b_{R}\right\rangle^{B}-C_{0}^{A} D_{1}^{B} t_{L}\left|c_{L} b_{R}\right\rangle^{B}, \\
& \left|\psi_{10,20}\right\rangle=\sqrt{2} r_{R} t_{R}\left(C_{3}^{A} D_{2}^{B} t_{L}\left|b_{L} c_{R}\right\rangle^{B}\right. \\
& \left.+C_{2}^{A} D_{3}^{B} r_{L}\left|c_{L} c_{R}\right\rangle^{B}\right) \text {, } \\
& \left|\psi_{10,02}\right\rangle=-\sqrt{2} r_{R} t_{R}\left(C_{3}^{A} D_{2}^{B} t_{L}\left|b_{L} c_{R}\right\rangle^{B}\right. \\
& \left.+C_{2}^{A} D_{3}^{B} r_{L}\left|c_{L} c_{R}\right\rangle^{B}\right) \text {, } \\
& \left|\psi_{01,20}\right\rangle=\sqrt{2} r_{R} t_{R}\left(C_{3}^{A} D_{2}^{B} r_{L}\left|b_{L} c_{R}\right\rangle^{B}\right. \\
& \left.-C_{2}^{A} D_{3}^{B} t_{L}\left|c_{L} c_{R}\right\rangle^{B}\right) \text {, } \\
& \left|\psi_{01,02}\right\rangle=\sqrt{2} r_{R} t_{R}\left(-C_{3}^{A} D_{2}^{B} r_{L}\left|b_{L} c_{R}\right\rangle^{B}\right. \\
& \left.+C_{2}^{A} D_{3}^{B} t_{L}\left|c_{L} c_{R}\right\rangle^{B}\right) \text {, } \\
& \left|\psi_{01,11}\right\rangle=\left(r_{R}^{2}-t_{R}^{2}\right)\left(C_{3}^{A} D_{2}^{B} r_{L}\left|b_{L} c_{R}\right\rangle^{B}\right. \\
& \left.-C_{2}^{A} D_{3}^{B} t_{L}\left|c_{L} c_{R}\right\rangle^{B}\right) \text {, } \\
& \left|\psi_{10,11}\right\rangle=\left(r_{R}^{2}-t_{R}^{2}\right)\left(C_{3}^{A} D_{2}^{B} t_{L}\left|b_{L} c_{R}\right\rangle^{B}\right. \\
& \left.+C_{2}^{A} D_{3}^{B} r_{L}\left|c_{L} c_{R}\right\rangle^{B}\right) \text {, } \\
& \left|\psi_{20,10}\right\rangle=\sqrt{2} r_{L} t_{L}\left(C_{3}^{A} D_{1}^{B} t_{R}\left|c_{L} b_{R}\right\rangle^{B}\right. \\
& \left.+C_{1}^{A} D_{3}^{B} r_{R}\left|c_{L} c_{R}\right\rangle^{B}\right) \text {, } \\
& \left|\psi_{02,10}\right\rangle=-\sqrt{2} r_{L} t_{L}\left(C_{3}^{A} D_{1}^{B} t_{R}\left|c_{L} b_{R}\right\rangle^{B}\right. \\
& \left.+C_{1}^{A} D_{3}^{B} r_{R}\left|c_{L} c_{R}\right\rangle^{B}\right) \text {, }
\end{aligned}
$$




$$
\begin{aligned}
\left|\psi_{20,01}\right\rangle= & \sqrt{2} r_{L} t_{L}\left(C_{3}^{A} D_{1}^{B} r_{R}\left|c_{L} b_{R}\right\rangle^{B}\right. \\
& \left.-C_{1}^{A} D_{3}^{B} t_{R}\left|c_{L} c_{R}\right\rangle^{B}\right), \\
\left|\psi_{02,01}\right\rangle= & \sqrt{2} r_{L} t_{L}\left(-C_{3}^{A} D_{1}^{B} r_{R}\left|c_{L} b_{R}\right\rangle^{B}\right. \\
& \left.+C_{1}^{A} D_{3}^{B} t_{R}\left|c_{L} c_{R}\right\rangle^{B}\right), \\
\left|\psi_{11,10}\right\rangle= & \left(r_{L}^{2}-t_{L}^{2}\right)\left(C_{3}^{A} D_{1}^{B} t_{R}\left|c_{L} b_{R}\right\rangle^{B}\right. \\
& \left.+C_{1}^{A} D_{3}^{B} r_{R}\left|c_{L} c_{R}\right\rangle^{B}\right), \\
\left|\psi_{11,01}\right\rangle= & \left(r_{L}^{2}-t_{L}^{2}\right)\left(C_{3}^{A} D_{1}^{B} r_{R}\left|c_{L} b_{R}\right\rangle^{B}\right. \\
& \left.-C_{1}^{A} D_{3}^{B} t_{R}\left|c_{L} c_{R}\right\rangle^{B}\right) .
\end{aligned}
$$

\section{Failed teleportation}

$$
\begin{gathered}
\left|\psi_{00,00}\right\rangle=C_{0}^{A} D_{0}^{B}\left|b_{L} b_{R}\right\rangle^{B}, \\
\left|\psi_{00,20}\right\rangle=\sqrt{2} C_{2}^{A} D_{2}^{B} r_{R} t_{R}\left|b_{L} c_{R}\right\rangle^{B}, \\
\left|\psi_{00,02}\right\rangle=-\sqrt{2} C_{2}^{A} D_{2}^{B} r_{R} t_{R}\left|b_{L} c_{R}\right\rangle^{B},
\end{gathered}
$$

$$
\begin{gathered}
\left|\psi_{20,00}\right\rangle=\sqrt{2} C_{1}^{A} D_{1}^{B} r_{L} t_{L}\left|c_{L} b_{R}\right\rangle^{B}, \\
\left|\psi_{02,00}\right\rangle=-\sqrt{2} C_{1}^{A} D_{1}^{B} r_{L} t_{L}\left|c_{L} b_{R}\right\rangle^{B}, \\
\left|\psi_{20,20}\right\rangle=2 C_{3}^{A} D_{3}^{B} r_{L} r_{R} t_{L} t_{R}\left|c_{L} c_{R}\right\rangle^{B}, \\
\left|\psi_{20,02}\right\rangle=-2 C_{3}^{A} D_{3}^{B} r_{L} r_{R} t_{L} t_{R}\left|c_{L} c_{R}\right\rangle^{B}, \\
\left|\psi_{02,02}\right\rangle=2 C_{3}^{A} D_{3}^{B} r_{L} r_{R} t_{L} t_{R}\left|c_{L} c_{R}\right\rangle^{B}, \\
\left|\psi_{02,20}\right\rangle=-2 C_{3}^{A} D_{3}^{B} r_{L} r_{R} t_{L} t_{R}\left|c_{L} c_{R}\right\rangle^{B}, \\
\left|\psi_{00,11}\right\rangle=C_{2}^{A} D_{2}^{B}\left(r_{R}^{2}-t_{R}^{2}\right)\left|b_{L} c_{R}\right\rangle^{B}, \\
\left|\psi_{11,00}\right\rangle=C_{1}^{A} D_{1}^{B}\left(r_{L}^{2}-t_{L}^{2}\right)\left|c_{L} b_{R}\right\rangle^{B}, \\
\left|\psi_{20,11}\right\rangle=\sqrt{2} C_{3}^{A} D_{3}^{B} r_{L} t_{L}\left(r_{R}^{2}-t_{R}^{2}\right)\left|c_{L} c_{R}\right\rangle^{B}, \\
\left|\psi_{02,11}\right\rangle=\sqrt{2} C_{3}^{A} D_{3}^{B} r_{L} t_{L}\left(t_{R}^{2}-r_{R}^{2}\right)\left|c_{L} c_{R}\right\rangle^{B}, \\
\left|\psi_{11,20}\right\rangle=\sqrt{2} C_{3}^{A} D_{3}^{B} r_{R} t_{R}\left(r_{L}^{2}-t_{L}^{2}\right)\left|c_{L} c_{R}\right\rangle^{B}, \\
\left|\psi_{11,02}\right\rangle=\sqrt{2} C_{3}^{A} D_{3}^{B} r_{R} t_{R}\left(t_{L}^{2}-r_{L}^{2}\right)\left|c_{L} c_{R}\right\rangle^{B}, \\
\left|\psi_{11,11}\right\rangle=C_{3}^{A} D_{3}^{B} r_{R} t_{R}\left(r_{L}^{2}-t_{L}^{2}\right)\left(r_{R}^{2}-t_{R}^{2}\right)\left|c_{L} c_{R}\right\rangle^{B} .
\end{gathered}
$$

[1] D. Gottesman and I. L. Chuang, Nature (London) 402, 390 (1999).

[2] M. A. Nielsen and I. L. Chuang, Quantum Computation and Quantum Information (Cambridge University Press, Cambridge, UK, 2000).

[3] C. H. Bennett, G. Brassard, C. Crepeau, R. Jozsa, A. Peres, and W. K. Wootters, Phys. Rev. Lett. 70, 1895 (1993).

[4] L.-M. Duan, J. I. Cirac, P. Zoller, and E. S. Polzik, Phys. Rev. Lett. 85, 5643 (2000).

[5] S. Bose, P. L. Knight, M. B. Plenio, and V. Vedral, Phys. Rev. Lett. 83, 5158 (1999).

[6] A. S. Parkins and H. J. Kimble, J. Opt. B: Quantum Semiclass. Opt. 2, 496 (1999).

[7] J. I. Cirac, P. Zoller, H. J. Kimble, and H. Mabuchi, Phys. Rev. Lett. 78, 3221 (1997).

[8] D. Bouwmeester, J.-W. Pan, K. Mattle, M. Eibl, H. Weinfurter, and A. Zeilinger, Nature (London) 390, 575 (1997).

[9] D. Boschi, S. Branca, F. De Martini, L. Hardy, and S. Popescu, Phys. Rev. Lett. 80, 1121 (1998).

[10] A. Furusawa, J. L. Sorensen, S. L. Braunstein, C. A. Fuchs, H. J. Kimble, and E. S. Polzik, Science 282, 706 (1998).

[11] Y.-H. Kim, S. P. Kulik, and Y. Shih, Phys. Rev. Lett. 86, 1370 (2001).

[12] I. Marcikic, H. de Riedmatten, W. Tittel, H. Zbinden, and N. Gisin, Nature (London) 421, 509 (2003).

[13] J.-W. Pan, S. Gasparoni, M. Aspelmeyer, T. Jennewein, and A. Zeilinger, Nature (London) 421, 721 (2003).

[14] R. Ursin, T. Jennewein, M. Aspelmeyer, R. Kaltenbaek, M. Lindenthal, P. Walther, and A. Zeilinger, Nature (London) 430, 849 (2004).

[15] J. F. Sherson, H. Krauter, R. K. Olsson, B. Julsgaard, K. Hammerer, I. Cirac, and E. S. Polzik, Nature (London) 443, 557 (2006).

[16] Y.-A. Chen, S. Chen, Z.-S. Yuan, B. Zhao, C.-S. Chuu, J. Schmiedmayer, and J.-W. Pan, Nat. Phys. (London) 4, 103 (2008).
[17] M. Riebe, H. Häffner, C. F. Roos, W. Hänsel, J. Benhelm, G. P. T. Lancaster, T. W. Körber, C. Becher, F. Schmidt-Kaler, D. F. V. James, and R. Blatt, Nature (London) 429, 734 (2004).

[18] M. D. Barrett, J. Chiaverini, T. Schaetz, J. Britton, W. M. Itano, J. D. Jost, E. Knill, C. Langer, D. Leibfried, R. Ozeri, and D. J. Wineland, Nature (London) 429, 737 (2004).

[19] S. Olmschenk, D. N. Matsukevich, P. Maunz, D. Hayes, L.-M. Duan, and C. Monroe, Science 323, 486 (2009).

[20] T. Di, A. Muthukrishnan, M. O. Scully, and M. S. Zubairy, Phys. Rev. A 71, 062308 (2005).

[21] X. Zou, J. Shu, and G. Guo, New J. Phys. 8, 246 (2006).

[22] B. P. Lanyon, M. Barbieri, M. P. Almeida, T. Jennewein, T. C. Ralph, K. J. Resch, G. J. Pryde, J. L. O'Brien, A. Gilchrist, and A. G. White, Nat. Phys. 5, 134 (2009); M. Neeley et al., Science 325, 722 (2009).

[23] M. S. Zubairy, Phys. Rev. A 58, 4368 (1998); M. Ikram, S.-Y. Zhu, and M. S. Zubairy, ibid. 62, 022307 (2000).

[24] F. Ciccarello, S. Bose, and M. Zarcone, Phys. Rev. A 81, 042318 (2010).

[25] Q. Zhang, A. Goebel, C. Wagenknecht, Y.-A. Chen, B. Zhao, T. Yang, A. Mair, J. Schmiedmayer, and J.-W. Pan, Nat. Phys. 2, 678 (2006).

[26] B. Julsgaard, A. Kozhekin, and E. S. Polzik, Nature (London) 432, 482 (2004).

[27] L. M. Duan, G. Giedke, J. I. Cirac, and P. Zoller, Phys. Rev. Lett. 84, 2722 (2000).

[28] K. S. Choi, H. Deng, J. Laurat, and H. J. Kimble, Nature (London) 452, 67 (2008).

[29] K. Bergmann, H. Theuer, and B. W. Shore, Rev. Mod. Phys. 70, 1003 (1998).

[30] M. O. Scully and M. S. Zubairy, Quantum Optics (Cambridge University Press, Cambridge, 1997).

[31] S. E. Harris, Phys. Today 50, 36 (1997).

[32] T. Di and M. S. Zubairy, J. Mod. Opt. 51, 2387 (2004).

[33] E. V. Moreva, G. A. Maslennikov, S. S. Straupe, and S. P. Kulik, Phys. Rev. Lett. 97, 023602 (2006). 
[34] Yu. I. Bogdanov, E. V. Moreva, G. A. Maslennikov, R. F. Galeev, S. S. Straupe, and S. P. Kulik, Phys. Rev. A 73, 063810 (2006).

[35] S.-Y. Baek, S. S. Straupe, A. P. Shurupov, S. P. Kulik, and Y.-H. Kim, Phys. Rev. A 78, 042321 (2008).

[36] T. Wilk, A. Gaëtan, C. Evellin, J. Wolters, Y. Miroshnychenko, P. Grangier, and A. Browaeys, Phys. Rev. Lett. 104, 010502 (2010).

[37] A. Gaëtan, Y. Miroshnychenko, T. Wilk, A. Chotia, M. Viteau, D. Comparat, P. Pillet, A. Browaeys, and P. Grangier, Nat. Phys. 5, 115 (2009).
[38] E. Urban, T. A. Johnson, T. Henage, L. Isenhower, D. D. Yavuz, T. G. Walker, and M. Saffman, Nat. Phys. 5, 110 (2009).

[39] M. S. Shahriar, J. A. Bowers, B. Demsky, P. S. Bhatia, S. Lloyd, P. R. Hemmer, and A. E. Craig, Opt. Commun. 195, 411 (2001).

[40] L. M. Duan and H. J. Kimble, Phys. Rev. Lett. 90, 253601 (2003).

[41] K. Hammerer, E. S. Polzik, and J. I. Cirac, Phys. Rev. A 72, 052313 (2005).

[42] R. Loudon, The Quantum Theory of Light, 3rd Ed. (Oxford University Press, Oxford, 2000). 